\title{
A Case of Ewing's Sarcoma of the Clavicle in Adults
}

\author{
Yijun Zhou, Zheng Tian, Akbar Yunus, Jiangtao Chen, Chong Wang, Leilei Xu, Xinghua Song*
}

Orthopaedic Department, The First Affiliated Hospital of Xinjiang Medical School, Urumqi, China.

Email: lizhouju357@126.com; gentelchan@126.com; "songxinghua19@163.com

Received April 17 , 2013; revised May 23 ${ }^{\text {rd }}, 2013$; accepted June $14^{\text {th }}, 2013$

Copyright (c) 2013 Yi Jun Zhou et al. This is an open access article distributed under the Creative Commons Attribution License, which permits unrestricted use, distribution, and reproduction in any medium, provided the original work is properly cited.

\begin{abstract}
The Clavicle is a rare primary site for Ewing sarcoma (ES). We report one case of patient with clavicular ES under our tracking and review the related literatures on management of this rare tumor. The patient was age of 35 years and was non metastatic at presentation. The patient received chemotherapy before and after the surgery. Claviculectomy was used. The patient had good functional outcomes with no pain of the shoulder at the follow-up of ten months. In conclusion, Ewing sarcoma of clavicle is a very rare tumor and different methods of surgical treatment can be used. Clavicle often can be resected without a need of reconstruction.
\end{abstract}

Keywords: Ewing’s Sarcoma; Adults; Clavicle

\section{Introduction}

Ewing sarcoma is the second most common childhood bone cancer [1]. Any bone can be affected, but it occurs more often in the femur and pelvis. Numerous series have been published, with the age at diagnosis usually younger than 30 years, especially 10 - 15 years. There is a 3:2 male predominance, and 95\% of Ewing sarcomas occurs in white patients $[2,3]$.

\section{Case Report}

We report a patient of Ewing's sarcoma of the clavicle, who is Kazak and presented at 35 years of age with a palpable mass over the right clavicle found by his parents 4 month prior to presentation. Physical examination revealed a $7 \times 6 \mathrm{~cm}$ palpable mass over the middle section of the right clavicle. It was firm and fixed to the underlying bone. No sign of infection was noted. The patient had full shoulder range of motion (ROM), with normal strength and sensation on the right upper extremity.

Laboratory investigation revealed Erythrocyte sedimentation rate of $19 \mathrm{~mm} / \mathrm{h}$, Alkaline phosphatase of 59.90 U/Land normal C-reaction protein normal. Plain radiographs demonstrated irregular center dissolved bony destruction, the decay sample destruction, increased bone mineral density around the shadow, periosteal reaction, and surrounding soft tissue swelling shadow in the Internal $1 / 3$ diaphysis of the clavicle extending to the ac-

\footnotetext{
*Corresponding author.
}

romial and sternal ends. However, the shoulder joint is normal (Figure 1). A bone scan increased uptake in the Internal 1/3 diaphysis of the right clavicle but there is no other area of abnormal uptake in the skeleton (Figure 2). Magnetic resonance imaging (MRI) documented a soft tissue mass surrounding the right clavicle as well as marrow involvement by the lesion involving nearly the entire clavicle (Figure 3). A chest computed tomography showed dissolved osseous destruction of obscure boundary with irregular bone cortex increased proliferation sclerosis, layered, Onion peel change or lace sample periosteal reaction in the Internal $1 / 3$ diaphysis of the right clavicle. The pathological fracture is located in the lateral 1/3 diaphysis of the right clavicle (Figure 4). A needle

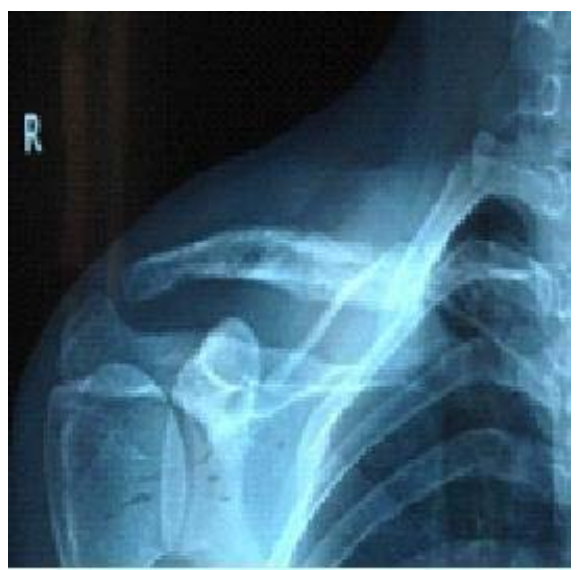

Figure 1. The anteroposterior X-ray before chemotherapy. 


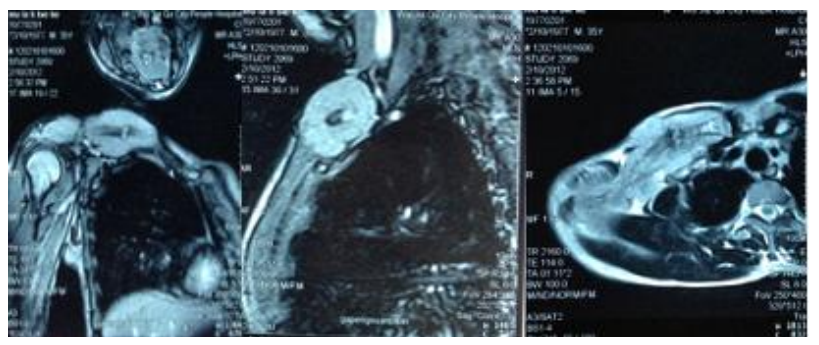

Figure 2. The MRI before chemotherapy.

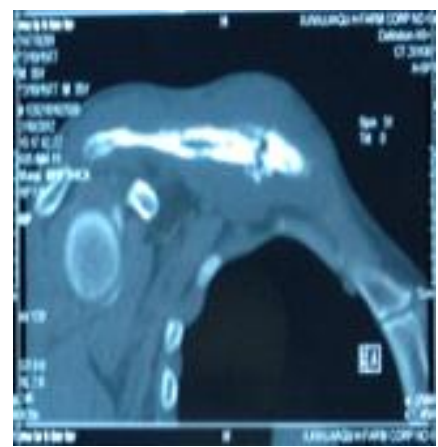

Figure 3. The CT before chemotherapy.

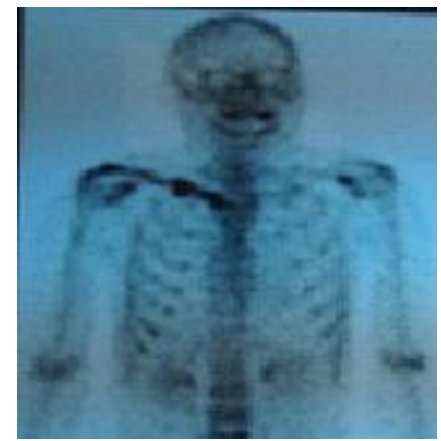

Figure 4. The ECT before chemotherapy.

biopsy revealed small round blue cells consistent with Ewing sarcoma (Figure 5).

The chemotherapy regimens adopted VAC + IE (vincristine, adriamycin, cyclophosphamide, ifosfamide, etoposide). The patient received three courses over 4 months in total. During chemotherapy period, the minimum white blood cells of the patient were $0.6 \times 10^{9} / \mathrm{L}$, and the patient appeared severe nausea and vomiting. White blood cells returned to normal, nausea and vomiting obviously alleviate after treatment with recombinant human granulocyte colony stimulating factor (rHuG-CSF) and metoclopramide. Following the response to the two courses of chemotherapy, the entire right clavicle was removed with a cuff of surrounding tissue.No reconstruction was performed, and there were no intraoperative complications. The patient's blood loss was $350 \mathrm{ml}$, and she tolerated the procedure well. No violation of the tumor mass occurred.

Reexamined MRI (Figure 5) demonstrates that: there is a diameter of 3.8 centimeters swelling lump in the Internal $1 / 3$ diaphysis of the right clavicle; the T1 showed equal signal intensity between muscle and mass center; the T2 showed inhomogeneous signal intensity enhancement in surrounding tissues of mass and high signal Mass center which indicated necrosis or cystic changes. Mass has obviously reduced compared before. Pathological fracture exists still. Finally, the patient underwent the procedure of the right clavicle total resection (Figure 6). Pathological results in the patient were morphologically consistent with a small round blue cell tumor (Figure 7)

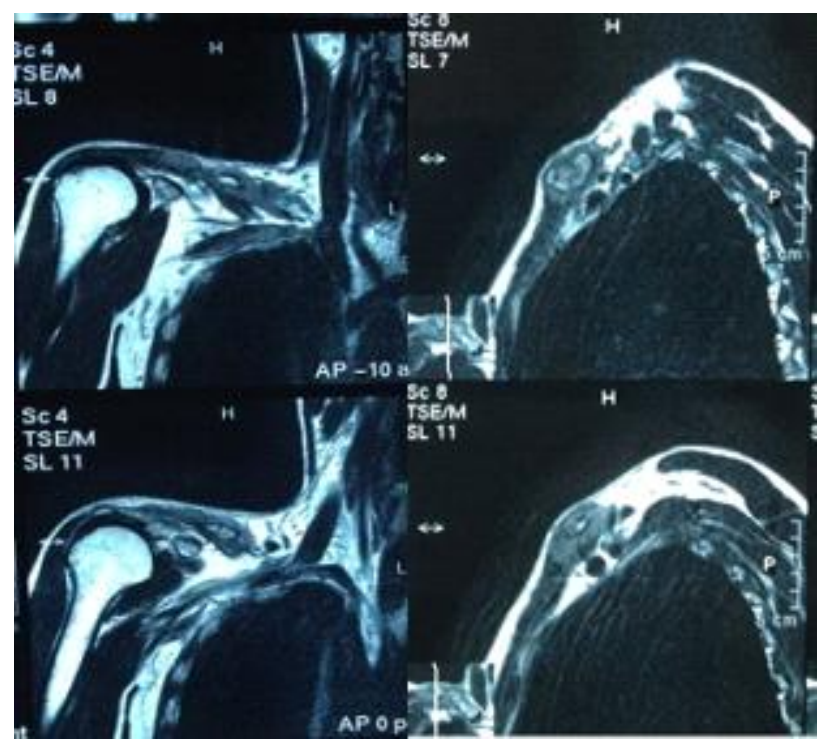

Figure 5. The MRI after chemotherapy.

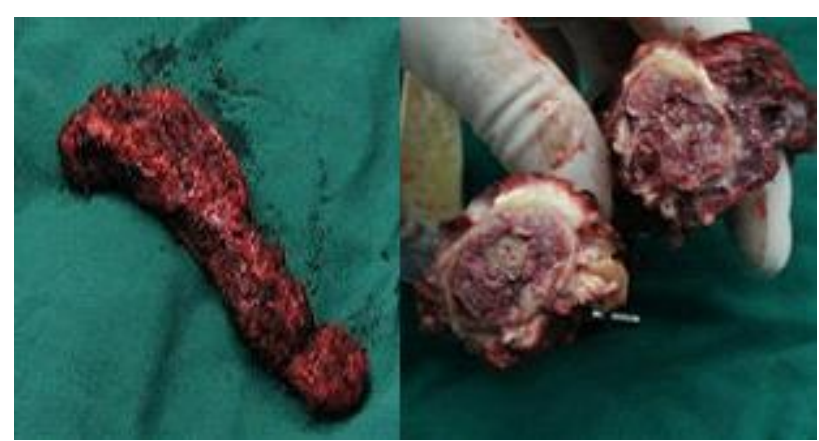

Figure 6. The intact clavicle of intraoperation.

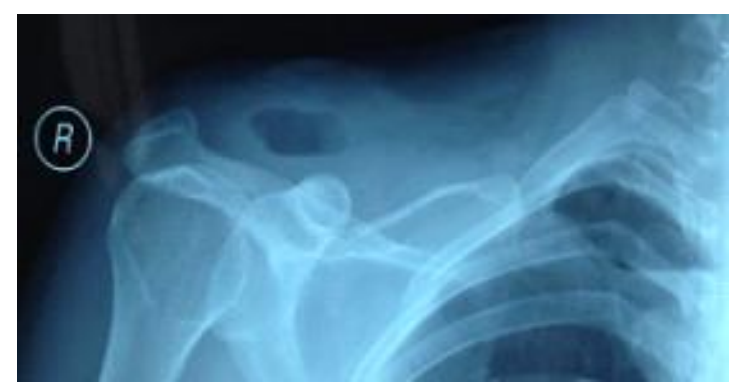

Figure 7. The anteroposterior X-ray of postoperative. 
which showed positivity for mic-2 (CD99) on immunohistochemistry.

Postoperatively, there was no surgical complication, and the wound healed well. She received two courses of postoperative chemotherapy over the next 4 months. He remains disease-free 10 months after surgery. Postoperatively, the patient's right arm was immobilized to his trunk for 3 days before allowing him to use his arm for daily activities. He has received no formal physical therapy. Functionally, he uses his right upper extremity normally, with strength equal to he contralateral nondominant side. He has intact vascular and neurological status of the right upper extremity and full range of motion of his right shoulder.

\section{Discussion}

Ewing sarcoma is a primary malignant tumor originally described by James Ewing in 1921. Immunocytochemical, cytogenetic, and molecular genetic investigations have suggested a neural crest origin for these malignant tumors [4]. Ewing sarcoma of the clavicle is an unusual lesion and there are few reports about the surgical in the current literature [5]. Ewing sarcoma is one of the most common bone tumors found in children and adolescents, second only to osteosarcoma. Other small round cell tumors found in this age group must be excluded before a diagnosis of Ewing sarcoma can be made. Histologically, it can appear similar to metastatic neuroblastoma, rhabdomyosarcoma, small cell osteosarcoma, and non-Hodgkin lymphoma. Recent molecular investigations have allowed more accurate pathologic diagnosis of Ewing sarcoma. Special stains such as mic-2 (CD99) help to differentiate it from other small round cell tumors. In addition, there is a clonal translocation in the malignant cells of Ewing sarcoma between the long arms of chromosome 11 and chromosome 22, which forms the fusion protein known as EWS-FLI1. This protein can be analyzed by using a small piece of tissue for polymerase chain reaction (PCR).

In Ewing sarcoma, the patient age is usually younger than 30 years (typically early adolescence). The male to female ratio is 3:2 [6]. It can involve almost any bone in the body; the most frequent sites of involvement are the femur, ilium, tibia, humerus, and fibula. In the thoracic cage, Ewing sarcoma predominates in the ribs, with less common or rare involvement of the scapula, clavicle, and sternum.

Ewing sarcoma has highly aggressive biological activity, which is reflected in its radiographic appearance, including osteolysis, cortical erosion, periostitis, and soft tissue masses. The bone destruction is generally permeative or moth eaten in appearance, and the periosteal response is often exuberant and may consist of multiple layers of new bone (laminated, onion skin, or onion peel pattern) or horizontally oriented thin osseous strands extending at a right angle to the parent bone (hair on end). On T1-weighted magnetic resonance images, tumors generally have signal intensity equal to or greater than that of muscles. Larger tumors appear as heterogeneous masses, frequently with evidence of hemorrhage or necrosis, whereas smaller ones tend to be more homogeneous. On T2-weighted images, the tumors tend to have inhomogeneous high signal intensity. Tumors show marked enhancement after intravenous administration of contrast material [7].

The patient is unusual for several reasons. Presentation of Ewing sarcoma during adulthood is rare. The clavicle is rarely involved in Ewing sarcoma. There is no such instances in the series by Maygarden et al. [8]. Other published series document fewer less than $5 \%$ of lesions in the clavicle [9]. Ewing sarcoma is also very rare among blacks and Asians; our patient is Kazak [10]. He is therefore most unusual in three ways: race, age, and affected bone. The case is instructive in alerting physicians that Ewing tumor is a possibility when an adult presents with an enlarging mass and typical radiographs, even if the adult does not fit the typical demographics for Ewing sarcoma. Finally, this brief report documents resection of the clavicle in a 35 years old adult with excellent residual function.

\section{REFERENCES}

[1] H. E. Grier, "The Ewing Family of Tumors. Ewing's Sarcoma and Primitive Neuroectodermal Tumors," Pediatric Clinics of North America, Vol. 44, No. 4, 1997, pp. 991-1004. doi:10.1016/S0031-3955(05)70541-1

[2] J. M. Kissane, F. B. Askin, M. Foulkes, et al., "Ewing's Sarcoma of Bone: Clinicopathologic Aspects of 303 Cases from the Intergroup Ewing's Sarcoma Study," Human Pathology, Vol. 14, No. 9, 1983, pp. 773-779. doi:10.1016/S0046-8177(83)80300-1

[3] K. K. Unni, "Dahlin's Bone Tumors: General Aspects and Data on 11,087 Cases," Lippincott-Raven, Philadelphia, 1996, pp. 249-261.

[4] S. M. Levine, R. E. Lambiase and C. N. Petchprapa, "Cortical Lesions of the Tibia: Characteristic Appearances at Conventional Radiography,” Radiographics, Vol. 23, No. 1, 2003, pp. 157-177. doi:10.1148/rg.231015088

[5] R. M. Juan, P. M. Juan, V. F. Rafael, et al., "Ewing Sarcoma of Clavicle in Children Report of 5 Cases,” Journal of Pediatric Hematology/Oncology, Vol. 11, No. 31, 2009, pp. 820-824.

[6] U. Tateishi, G. W. Gladish, M. Kusumoto, et al., "Chest Wall Tumors: Radiologic Findings and Pathologic Correlation, Part 2. Malignant Tumors,” Radiographics, Vol. 23, No. 6, 2003, pp. 1491-1508. doi:10.1148/rg.236015527

[7] M. Amit, M. Vinod, B. Permeet, et al., "Sunray Appearance on Sonography in Ewing Sarcoma of the Clavicle," 
Journal of Ultrasound in Medicine, Vol. 29, No. 3, 2010, pp. 493-495.

[8] E. J. Burgert, M. E. Nesbit, L. A. Garnsey, et al., "Multimodal Therapy for the Management of Nonpelvic, Localized Ewing's Sarcoma of Bone: Intergroup Study IESSII,” Journal of Clinical Oncology, Vol. 8, No. 9, 1990, pp. 1514-1524.

[9] R. M. Wilkins, D. J. Pritchard, E. O. Burgert, et al., "Ew- ing's Sarcoma of Bone: Experience with 140 Patients," Cancer, Vol. 58, No. 11, 1986, pp. 2551-2555. doi:10.1002/1097-0142(19861201)58:11<2551::AID-CN CR2820581132>3.0.CO;2-Y

[10] D. M. Parkin, C. A. Stiller and J. Nectoux, "International Variations in the Incidence of Childhood Bone Tumors," International Journal of Cancer, Vol. 53, No. 3, 1993, pp. 371-376. doi:10.1002/ijc.2910530305 